\title{
Simulation on Spacecraft Formation Flight and Formation Reconfiguration
}

\author{
Chao Li Ye Yan and Yue-neng Yang \\ National University of Defense Technology, College of Aerospace Science and Technology, 410073, Changsha, China
}

\begin{abstract}
Spacecraft formation flight refers to two or more spacecraft according to a certain formation or arrangement of flight, it has important application value for deep spatial exploration, spatial science experiment, ground investigation and military and so. This paper studies the spacecraft formation flight design and formation reconfiguration based on STK. Firstly, the Clohessy-Wiltshire (CW) equation is used to describe the relative motion of the near-circular orbit and deduce the relative orbital dynamics model. Then, based on the dynamic method of the $\mathrm{CW}$ equation, the spatial circular formation is designed and the STK is applied to simulate it. Finally, based on the above formation, a simple multi-impulse formation reconfiguration is performed and the simulation test is verified by STK. The simulation results show that the absolute error of orbital elements of the spacecraft is calculated by the relative orbital dynamics model is less than $10^{-5}$, and the expected formation can be completed under the condition of two-body environment, and the feasibility of simple multi-pulse formation reconfiguration is proved successfully.
\end{abstract}

\section{Introduction}

Spacecraft formation flight technology refers to the multi-spacecraft constructed cluster, the system is closely coordinated with each other, so as to form a unified organic whole, and the performance goes beyond the traditional single spacecraft system [1]. Relative motion dynamics, control and formation design optimization are essential for formation flight technology, so considering the design and reconfiguration attracted the attention of many scholars [2].

Pan[2]emphatically expounds the development of spacecraft formation flight and its key technology and introduced that spacecraft formation flight play an important role for GPS and relative navigation technology, it laid a solid foundation for the research work of many scholars. Abbott[3] present a method for finding a minimum-power dipole solution for a given set of desired forces, which does not rely on arbitrary parameterizations as have prior approaches, and the structure enables further analysis of numerical conditioning and convergence. $\mathrm{Wu}$ [4]designed a linear quadratic control algorithm based on HILL equation dynamics model to verify the feasibility of that control algorithm and its simulation realization method, and laid a certain foundation for formation reconfiguration. Seo[5] investigates Collision avoidance strategies for multiple UAVs (Unmanned Aerial Vehicles) based on geometry and Numerical simulations are performed to demonstrate the performance of the proposed strategies. Jiang[6]studied two-point boundary value problems of relative motion, the algebraic method was used to study the geometric properties of the relative orbit and provided a reliable mathematical basis for the formation design. Meng [1] further studied the theory and method involved in the two-body orbit formation of relative motion dynamics and formation design, for the relative navigation, control and formation optimization design provides a reliable theoretical basis.

Aiming at the multi-purpose and multi-tasking of spacecraft formation, design the formation becomes the key and foundation of the task. Therefore, the spatial circular formation is designed based on the $\mathrm{C}-\mathrm{W}$ equation dynamic method and by applying simple multi-impulse to achieve the formation reconfiguration, and the simulation test is verified and analyzed based on STK [6].

\section{Design Spatial Circular Formation Based on Dynamic Equation}

\subsection{Spatial circular formation}

The spatial circular formation refers to the around spacecraft with reference spacecraft as the center and the relative motion trace is circle orbit. In addition, the phase difference between the three spacecraft is $120^{\circ}$ and uniform distribution on the spatial circle.

The orbital elements of the reference spacecraft as follows: 
Table 1. The orbital elements of the reference spacecraft.

\begin{tabular}{|c|c|c|c|c|c|c|}
\hline & $a / \mathrm{km}$ & $e$ & $i^{/}$ & $\Omega /{ }^{\circ}$ & $\omega /^{\circ}$ & $M /^{\circ}$ \\
\hline $\begin{array}{l}\text { Reference } \\
\text { Spacecraft }\end{array}$ & 7000 & 0 & 60 & 10 & 20 & 30 \\
\hline
\end{tabular}

\subsection{Formation design based on $\mathrm{C}-\mathrm{W}$ equation}

Model design flow chart as follows:

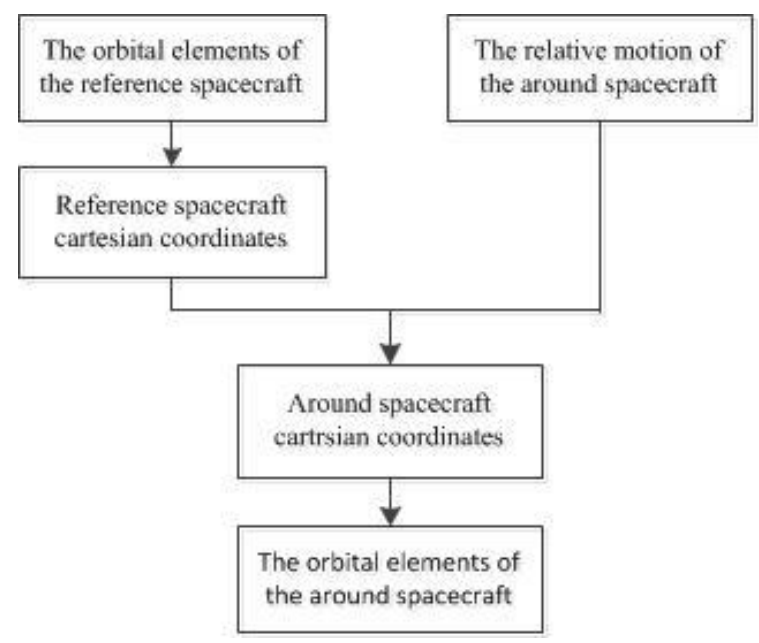

Figure 1. Model design flow.

Assuming that the earth is a homogeneous sphere and regardless of any perturbation factor, $[x, y, z]^{T}$ is the relative position vector of the spacecraft relative to the reference spacecraft, by solving the $\mathrm{CW}$ equation ${ }^{[1]}$ :

$$
\left\{\begin{array}{l}
\ddot{x}=2 n \dot{y}+3 n^{2} x \\
\ddot{y}=-2 n \dot{x} \\
\ddot{z}=-n^{2} z
\end{array}\right.
$$

The analytical form of equation solution can be obtained:

$$
\left\{\begin{aligned}
x(t) & =\frac{\dot{x}_{0}}{n} \sin (n t)+\left(-3 x_{0}-\frac{2 \dot{y}_{0}}{n}\right) \cos (n t)+2\left(2 x_{0}+\frac{\dot{y}_{0}}{n}\right) \\
y(t) & =2\left(3 x_{0}+\frac{2 \dot{y}_{0}}{n}\right) \sin (n t)+\frac{2 \dot{x}_{0}}{n} \cos (n t)-3\left(2 n x_{0}+\dot{y}_{0}\right) t \\
& +\left(-\frac{2 \dot{x}_{0}}{n}+y_{0}\right) \\
z(t) & =\frac{\dot{z}_{0}}{n} \sin (n t)+z_{0} \cos (n t)
\end{aligned}\right.
$$

$x_{0}, \mathrm{y}_{0}, \mathrm{z}_{0}$ is the initial state of the relative motion of a given reference frame.

If the formula $\dot{y}_{0}=-2 n x_{0}$ can be satisfied, the drift will be eliminated so that relative motion formation is stable [7-8]. $n=\sqrt{\mu / a_{\text {ref }}}$ is the average orbital angular velocity of reference spacecraft and $\mu$ is geocentric gravitational constant. Further processing the stable relative motion solution, get the simpler form:

$$
\left\{\begin{array}{l}
x=-A \cos (n t+\theta) \\
y=2 A \sin (n t+\theta)+L \\
z=-B \cos (n t+\varphi)
\end{array}\right.
$$

among them:

$$
\begin{aligned}
& A=-\sqrt{x_{0}^{2}+\left(\dot{x}_{0} / n\right)^{2}},\left\{\begin{array}{l}
\cos \theta=-x_{0} / A \\
\sin \theta=\dot{x}_{0} /(n A)
\end{array}\right. \\
& B=-\sqrt{z_{0}^{2}+\left(\dot{z}_{0} / n\right)^{2}} \\
& \left\{\begin{array}{l}
\cos \varphi=-z_{0} / A \\
\sin \varphi=\dot{z}_{0} /(n A)
\end{array} ; L=-\frac{2 \dot{x}_{0}}{n}+y_{0}\right.
\end{aligned}
$$

$A, \theta, B, \varphi, L$ are the amplitude and phase of the elliptical orbital plane, the amplitude and phase along the normal direction of the orbital surface, and the position of the ellipse center in the reference frame. On this basis, considering that the center is located at the origin of reference frame, it is necessary to meet the constraints $L=-\frac{2 \dot{x}_{0}}{n}+y_{0}=0$.

From the analysis, it can be:

$$
\left\{\begin{array}{l}
\cos \theta=-x_{0} / A \\
\sin \theta=\dot{x}_{0} /(n A) \\
y_{0}=\frac{2 \dot{x}_{0}}{n} \\
\dot{y}_{0}=-2 n x_{0} \\
\cos \varphi=-z_{0} / B \\
\sin \varphi=\dot{z}_{0} /(n B)
\end{array}\right.
$$

The relative motion state of around spacecraft relative to reference spacecraft $x_{0}, y_{0}, z_{0}, \dot{x}_{0}, \dot{y}_{0}, \dot{z}_{0}$ is obtained. The formation design ultimately get the initial motion state of each spacecraft in the formation, the Cartesian coordinate state of the geocentric inertia system, or the orbital elements. Therefore, the formation design of the spacecraft formation based on the $\mathrm{C}-\mathrm{W}$ equation is as follows:

(1). According to the orbital elements of the reference spacecraft calculate the position vector $R_{c}$ and velocity vector $V_{c}$ of the reference spacecraft in the geocentric inertial system.

(2).According to the expected radius, the around spacecraft in the orbital coordinate system relative to the reference spacecraft position $r$, speed $v$ is calculated.

(3).Can be converted by the conversion matrix $Q=Q_{z}[-\Omega] Q_{x}[-i] Q_{z}[-u] \quad\left(Q_{j}(\theta)\right.$ is the elementary transformation matrix that turns $\theta$ around the $\mathrm{j}$ axis), the around spacecraft in the orbit coordinate system relative to the reference spacecraft position $r$, the speed $v$ through the coordinate conversion to the inertial coordinate system;

(4).The position $R_{h}$ and velocity $V_{h}$ of the around 
spacecraft in the geocentric inertial system can be obtained by adding the sum of the position $r$, vector $v$ in step (3) and the position $R_{c}$, vector $V_{c}$ of the reference spacecraft.

(5).According to the position $R_{h}$ and velocity $V_{h}$ of the around spacecraft, the orbital elements of the spacecraft can be calculated.

\subsection{Simulation results and analysis}

Based on the above mathematical model, can calculate the orbital elements of the three around spacecraft, and then according to the following six graphs can be analyzed: The semi-major axis $a$ of ellipse of the around spacecraft is less than 50 meters. Eccentricity $e$ difference is less than $10^{-10}$; Inclination $i$ difference is less than $0.1^{\circ}$. The right ascension of ascending node $\Omega$ is less than $0.1^{\circ}$. True anomaly $\omega$ are $0^{\circ}, 120^{\circ}$ and $240^{\circ}$ respectively. The Argument of latitude $M$ are $50^{\circ}, 290^{\circ}$ and $170^{\circ}$ respectively.

The orbital elements of the around spacecraft are shown in Table 2:

Combined with orbital elements of the above four spacecraft, STK is used to make the simulation and the following simulation results are obtained.

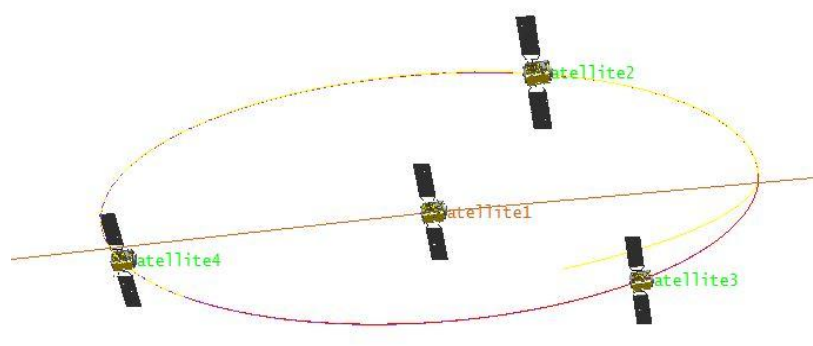

Figure 2. The spatial circular formation obtained by STK.
When the results of the mathematical model are calculated and visual simulation is made by STK, four spacecraft formation is the spatial circular formation, which that three around spacecraft was $120^{\circ}$ phase difference. It can be seen that the $\mathrm{C}-\mathrm{W}$ equation is used to design formation, and the correctness of the mathematical model and simulation experiment is proved.

\section{Formation reconfiguration based on simple multi-impulse}

\subsection{Carpe formation}

Carpe formation refers to the around spacecraft take reference spacecraft as center, one of the around spacecraft and reference stars are coplanar, and the around one do an elliptical motion. The relative motion of the other two around spacecraft is perpendicular to the orbit plane of reference spacecraft, and make a linear oscillatory motion, phase difference between the three spacecraft is $90^{\circ}$.

\subsection{The condition of formation reconstruction}

The initial phases of the three spacecraft in the spatial circle are: $0^{\circ}, 120^{\circ},-120^{\circ}$. It is known that the motion equations of the three around spacecraft relative to the reference spacecraft are:

$$
\left\{\begin{array}{l}
x=-500 \cos (n t+\theta) \\
y=1000 \sin (n t+\theta) \\
z=-500 \sqrt{3} \cos (n t+\theta)
\end{array}\right.
$$

According to the orbital elements of the reference spacecraft in the spatial circle, the orbital elements of Sat_1', Sat_2' and Sat_3'in the CarPe formation can be calculated. The calculation results are shown in the table 3 .

In order to reconstruct the CarPe formation, the three around spacecraft are maneuvered by adding a simple multi-impulse so that it can be realized.

Table 2. The orbital elements of the reference spacecraft and three around spacecraft.

\begin{tabular}{|c|c|c|c|c|c|c|}
\hline & $a / \mathrm{km}$ & $e$ & $i /^{\circ}$ & $\Omega /{ }^{\circ}$ & $\omega /^{\circ}$ & $M / /^{\circ}$ \\
\hline ref & 7000 & 0 & 60 & 10 & 20 & 30 \\
\hline Sat_1 & 7000.000072 & $7.14286 \times 10^{-5}$ & 59.9946 & 10.0053 & 49.9974 & 0 \\
\hline Sat_2 & 7000.000232 & $7.14286 \times 10^{-5}$ & 60.0067 & 10.0028 & 289.999 & 120 \\
\hline Sat_3 & 7000.000232 & $7.14286 \times 10^{-5}$ & 59.9988 & 9.992 & 170.004 & 240 \\
\hline
\end{tabular}

Table 3. The orbital elements of the reference spacecraft and three around spacecraft.

\begin{tabular}{|c|c|c|c|c|c|c|}
\hline & $\mathrm{a} / \mathrm{km}$ & $\mathrm{e}$ & $\mathrm{i} /{ }^{\circ}$ & $\Omega /{ }^{\circ}$ & $\omega /{ }^{\circ}$ & $\mathrm{M} /{ }^{\circ}$ \\
\hline ref & 7000 & 0 & 60 & 10 & 20 & 30 \\
\hline
\end{tabular}




\begin{tabular}{|c|c|c|c|c|c|c|}
\hline Sat_1' & 7000 & $7.14286 \times 10^{-5}$ & 60 & 10 & 20 & $7.105 \times 10^{-15}$ \\
\hline Sat_2' & 7000 & 0 & 60.0026 & 10.0036 & 20.0006 & 30 \\
\hline Sat_3' & 7000 & 0 & 59.9974 & 9.99638 & 19.9994 & 30 \\
\hline
\end{tabular}

$M_{s}$ once:

\subsection{Design elliptical formation on orbit plane}

An impulse is applied to the Sat_1, which is maneuvered to perform elliptical flight relative to the reference spacecraft on the spacecraft's orbital plane. Since this maneuver is a coplanar orbit maneuver, it is necessary to apply impulse to the normal direction to eliminate the relative motion of the vertical direction. $\Delta V_{1}$ is impulse which applied to the spacecraft, the relative motion equation as follows:

$$
\left\{\begin{array}{l}
x=-500 \cos f \\
y=1000 \sin f \\
z=-500 \sqrt{3} \cos f+\frac{\Delta V_{1}}{n} \sin \left(f-f_{o 1}\right)
\end{array}\right.
$$

To eliminate the relative motion of the vertical direction of orbital plane (that is the $\mathrm{Z}$ direction), according to the above equation:

$$
\left\{\begin{array}{l}
\frac{\Delta V_{1}}{n} \sin f_{o 1}+500 \sqrt{3}=0 \\
\frac{\Delta V_{1}}{n} \cos f_{o 1}=0
\end{array}\right.
$$

The available $f_{o 1}$ solutions are $f_{o 1}=k \pi+\frac{1}{2} \pi, k=0,1,2, \ldots . n$, then $\Delta V_{1}=500 \sqrt{3} n \approx 0.93358 \mathrm{~m} / \mathrm{s}$.

From the above parameters:

$$
\begin{gathered}
\left\{\begin{array}{c}
\Delta V_{1}=n B_{c}=n\left[B_{f}^{2}+B_{0}^{2}-2 B_{f} B_{0} \cdot \cos \left(\eta_{f}-\eta_{0}\right)\right]^{1 / 2} \\
M_{1}=-\eta_{0}-\eta_{c}+\frac{\pi}{2}
\end{array}\right. \\
\left\{\begin{array}{c}
\mathrm{n} \eta_{c}=\frac{B_{f} \sin \left(\eta_{f}-\eta_{0}\right)}{B_{c}} \\
\cos \eta_{c}=\frac{B_{f} \cos \left(\eta_{f}-\eta_{0}\right)-B_{0}}{B_{c}} \\
n=\sqrt{\mu / a^{3}} \quad t_{1}=M_{1} / 57.3 n
\end{array}\right.
\end{gathered}
$$

The time of the impulse $t_{1}$ can be obtained.

\subsection{Design oscillation on the vertical direction of spacecraft orbital plane}

First, it is necessary to eliminate the shifting $\Delta L$ between the formation center of the spatial circle and the formation center of CarPe. By applying a radial control impulse

$$
\left\{\begin{array}{l}
\Delta V_{s}=-\frac{1}{2} n \Delta L \\
M_{s}=-\eta_{0}+\frac{\pi}{2} \\
t_{s}=M_{s} / 57.3 n
\end{array}\right.
$$

Substituting the formation parameters, the amount of the impulse along the normal direction and the time of the application can be applied.

And then three times along the trace control impulse would be applied:

$$
\begin{aligned}
& \left\{\begin{array}{r}
\Delta V_{T 1}=\frac{n}{8} A_{c}=\frac{n}{8}\left[A_{f}^{2}+A_{0}^{2}-2 A_{f} A_{0} \cdot \cos \left(\theta_{f}-\theta_{0}\right)\right]^{1 / 2} \\
M_{01 T}=-\theta_{0}-\theta_{c}
\end{array}\right. \\
& \sin \theta_{c}=\frac{A_{f} \sin \left(\theta_{f}-\theta_{0}\right)}{A_{c}} \quad \cos \theta_{c}=\frac{A_{f} \cos \left(\theta_{f}-\theta_{0}\right)-A_{0}}{A_{c}} \\
& \begin{array}{ll}
\Delta V_{T 2}=-2 \Delta V_{T 1}=-2 \Delta V_{T 3} \quad n=\sqrt{\mu / a^{3}} & t=M_{T} / 57.3 n
\end{array} \\
& M_{02 T}=M_{01 T}+\pi \\
& M_{03 T}=M_{02 T}+\pi
\end{aligned}
$$

The initial formation parameters of Sat_2'and Sat_2 are obtained from the previous contents:

$$
\left\{\begin{array}{c}
A_{0}=-\frac{1}{2} \quad B_{0}=\frac{\sqrt{3}}{2} \quad \theta_{0}=120^{\circ} \quad \eta_{0}=120^{\circ} \\
A_{f}=0 \quad B_{f}=1 \quad \theta_{f}=0^{\circ} \quad \eta_{f}=0
\end{array}\right.
$$

Substituting the formation parameters to obtain the amount of impulse along the trace and the time of the application.

Finally, a control impulse $M_{W}$ is applied once along normal direction of the orbital plane:

$$
\begin{aligned}
& \left\{\begin{array}{c}
\Delta V_{W}=n B_{c}=n\left[B_{f}^{2}+B_{0}^{2}-2 B_{f} B_{0} \cdot \cos \left(\eta_{f}-\eta_{0}\right)\right]^{1 / 2} \\
M_{W}=-\eta_{0}-\eta_{c}+\frac{\pi}{2}
\end{array}\right. \\
& \sin \eta_{c}=\frac{B_{f} \sin \left(\eta_{f}-\eta_{0}\right)}{B_{c}} \quad \cos \eta_{c}=\frac{B_{f} \cos \left(\eta_{f}-\eta_{0}\right)-B_{0}}{B_{c}} \\
& n=\sqrt{\mu / a^{3}} \quad t=M_{W} / 57.3 n
\end{aligned}
$$

Substituting the formation parameters, the amount of the impulse along the normal direction and the time of the application are obtained. Calculating the amount of the applied impulse and the time to get the following two tables: 
Table 4. Impulse amount during reconfiguration $(\mathrm{m} / \mathrm{s})$.

\begin{tabular}{|c|c|c|c|c|c|}
\hline & $\begin{array}{c}\text { Frist trace } \\
\text { direction }\end{array}$ & $\begin{array}{c}\text { Second trace } \\
\text { direction }\end{array}$ & $\begin{array}{c}\text { Third trace } \\
\text { direction }\end{array}$ & $\begin{array}{c}\text { Normal } \\
\text { direction }\end{array}$ & $\begin{array}{c}\text { Radial } \\
\text { direction }\end{array}$ \\
\hline Sat_1 & 0 & 0 & 0 & 0.9336 & 0 \\
\hline Sat_2 & 0.04716 & -0.09433 & 0.047163 & 1.426070 & -0.1617 \\
\hline Sat_3 & 0.0876 & -0.1752 & 0.0876 & 1.4261 & 0.1617 \\
\hline
\end{tabular}

Table 5. Apply the time interval during reconfiguration (s).

\begin{tabular}{|c|c|c|c|c|c|}
\hline & $\begin{array}{c}\text { Frist trace } \\
\text { direction }\end{array}$ & $\begin{array}{c}\text { Second trace } \\
\text { direction }\end{array}$ & $\begin{array}{c}\text { Third trace } \\
\text { direction }\end{array}$ & $\begin{array}{c}\text { Normal } \\
\text { direction }\end{array}$ & $\begin{array}{c}\text { Radial } \\
\text { direction }\end{array}$ \\
\hline Sat_1 & 0 & 0 & 0 & 4371.3872 & 0 \\
\hline Sat_2 & 1457.1291 & 2914.2581 & 2914.2581 & 1633.4966 & 5342.8065 \\
\hline Sat_3 & 1457.1291 & 2914.2581 & 2914.2581 & 1280.7615 & 3399.9678 \\
\hline
\end{tabular}

\subsection{Simulation results and analysis}

According to the above results that the spatial circular formation can complete the formation reconfiguration successfully, and the simulation process of the reconfiguration as follows:

(1) The state before reconfiguration:

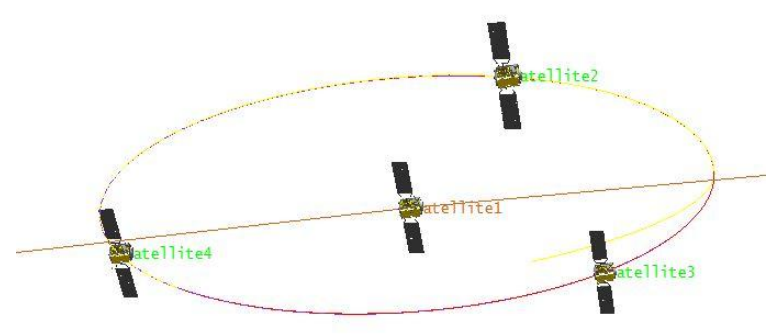

Figure 3. The state before reconfiguration.

(2) During the process of orbital maneuver, the relative motion between the spacecraft is:

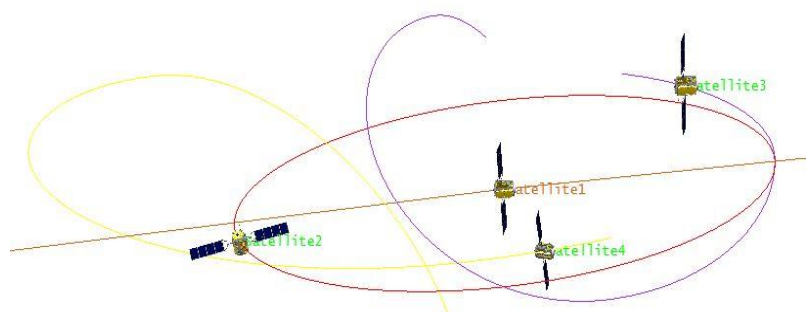

Figure 4. State of motion during reconfiguration(1).

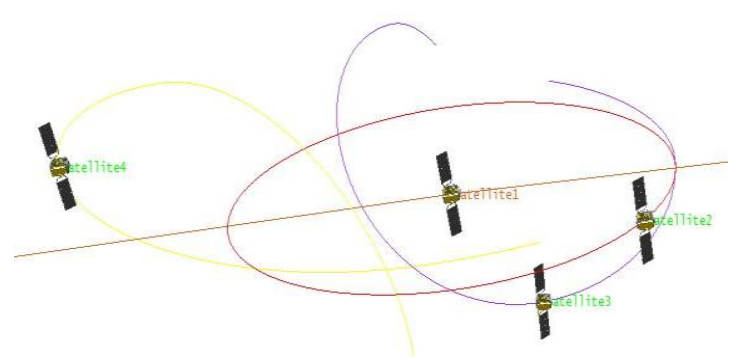

Figure 5. State of motion during reconfiguration(2).

(3) Maneuver is completed, Carpe formation as shown:

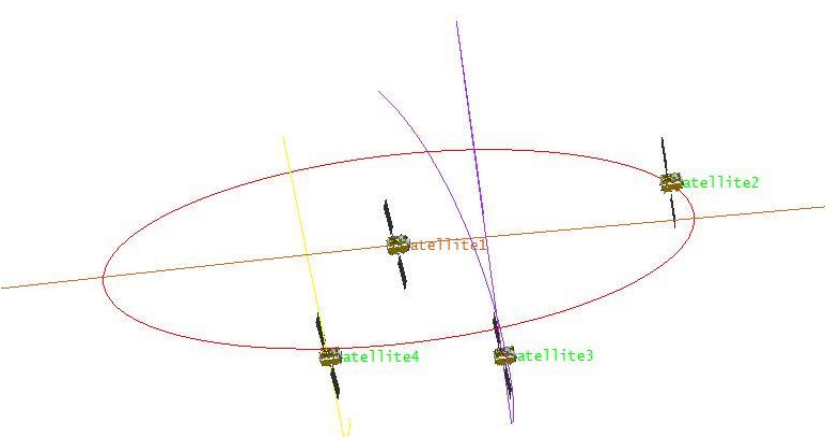

Figure 6. The state after reconfiguration.

According to the goal of the formation reconfiguration, taking advantage of the results which are calculated by the mathematical model to carrying out visualization simulation by STK, the formation of the four spacecraft is changed from the spatial circle formation by applying multiple impulses (Table 4, Table 5), gradually become the CarPe formation, the above analysis process can successfully verify the correctness of the mathematical model and the simple multi-impulse formation reconfiguration. 


\section{Conclusion}

Based on the characteristics of spacecraft formation flight, this paper designs the spatial circular formation, and takes advantage of the simple multi-impulse to complete the formation reconfiguration task, which provides a more effective solution for the complicated and changeable tasks in spatial. The whole formation system cooperates with each other to realize the multi-purpose and multi-tasking of the spacecraft formation network ${ }^{[9-10]}$, which can be applied to the formation design of the specific task.

\section{References}

1. Meng Yunhe. Spacecraft formation flight introduction [M], Beijing: National defense industry press, 2014.

2. Pan Keyan. The development of spacecraft formation flight and its key technology [J], Telemetry remote control, 24 (5): 9-15,2003.

3. Jake J.Abbott, Joseph B. Brink, and Braxton Osting. Computing Minimum-Power Dipole Solutions for Interdipole Forces Using Nonlinear Constrained Optimization With Application to Electromagnetic Formation Flight[J], IEEE Robotics and automation letters,2(2) :1008-1014,2017.
4. Wu Xia, Zheng Jianhua. Maintain Control and simulation of formation flight based on STK [J], Computer simulation, 24 (3): 66-68,2007.

5. Joongbo Seo, Youdan Kim.Collision Avoidance Strategies for Unmanned Aerial Vehicles in Formation Flight[J],IEEE Transactions on aerospace and electronic systems,0018-9251(c),2016.

6. Jiang Fanghua. Research on the relative motion of spacecraft formation flight[D], Beijing: Tsinghua University, 2009.

7. Zhang Jinwu. Research on unmanned aerial vehicle formation flight technology[J], Ship electronic engineering,,35 (8): 9-12,2015.

8. Wu Yun-hua, Cao Xi-bin. Research on autonomous relative navigation algorithm of formation flight spacecraft $[\mathrm{J}]$; Journal of Harbin institute of technology, 39 (3): 354-358,2007.

9. Gao Youtao. Research on the modeling and control of flight dynamics of spacecraft formation[D], Nanjing: Nanjing university of aeronautics and astronautics, 2010.

10. Yu Ping, Zhang Honghua. Typical modal and formation control method for elliptical orbit formation flight $[\mathrm{J}]$, Journal of astronautics,,26 (1): 7-12,2005 